\title{
Mobile Device Considerations for Supply Chain and ERP Related Systems
}

\author{
Byron Clemens, Teuta Cata and Gary Hackbarth
}

Northern Kentucky University, Highland Heights, USA

\begin{abstract}
The increased mobility of users and rapid enhancements to "hand held" technology suggest leveraging mobile technology to enhance timely transaction handling and data collection, as well as access robust tools for management decision making in supporting Supply Chain Management (SCM), Enterprise Resource Planning (ERP) and other complimentary systems. Though technology options are prevalent, applications must be developed for fit and viability on mobile devices which do not deter them from needed system functionality. Not only must internal application requirements address the mobility needs of its users, but interaction with external entities (e.g. suppliers) must be considered, as well. This paper reviews the history of devices used for Supply Chain Management and ERP applications through transition to mobile devices, as well as suggesting considerations for mobility and reachability and other factors that influence the utilization of newer technologies.
\end{abstract}

Keywords: Mobile Supply Chain, Mobile User Interface, Cloud Computing for Supply Chain.

\section{Introduction}

Mobile commerce, or m-commerce, has been described as the next generation of $\mathrm{e}$ commerce. In a broader sense, it refers to any transaction taking place, either directly or indirectly, through the use of a mobile or handheld computing device, using wireless technologies and telecommunication networks. The most significant feature of $\mathrm{m}$ commerce and its related technologies is the ability to be on the move, largely independent of time and location. Key characteristics are "mobility" (or portability) and "reachability". Related to these characteristics is the business value of enhancing traditional e-commerce in terms of product and service localization, personalization, heightened capability for accessing services ubiquitously, instant connectivity andconvenience (Liang, Huang, Yeh, \& Lin, 2007). With the competitive pressure and advancement in mobile networks and technologies, the popularity of mobile business-to-business (B2B), employee-to-business (E2B) and business-toemployee (B2E) solutions have continued to rapidly grow (Kurbel, Jankowska, \& Nowakowski, 2006).

From the perspective of e-commerce for Supply Chain Management (SCM) and Enterprise Resource Planning (ERP) and related systems, the most significant advancement of mobile technology has not been the devices themselves, but rather the ability to integrate in real-time with the "heavy hitter" backend systems, instead of continuing the legacy system attribute of delayed, batchprocessing (Shacklett, 2010). The result has been increased real-time interaction between companies, their employees, partners in the supply chain as well as customers, with enhanced operating

Copyright (C) 2012 Byron Clemens, Teuta Cata and Gary Hackbarth. This is an open access article distributed under the Creative Commons Attribution License unported 3.0, which permits unrestricted use, distribution, and reproduction in any medium, provided that original work is properly cited. Contact author: Teuta Cata E-mail: catat@nku.edu 
efficiency and provisions for new opportunities in providing customer service (Shankar, 2002). From a SCM perspective, $\mathrm{m}$-commerce provides significant impact in e-procurement, materials handling, warehousing, inventory management, logistics and fulfillment, asset tracking as well as sales and field force automation and dispatch management (Doolin \& Al Haj Ali, 2008).

All of this is enhanced by the technological advancements that make integration timely and seamless to the end-user. 3G broadband wireless technology and the more recent $4 \mathrm{G}$ services are providing bandwidth to handle multiple functions simultaneously. Mobile users can quickly collect and interact with data not readily available outside "brick-andmortar" locations, including video, pictures and graphics, with clarity, speed and mobility not available with earlier generations of technology. Businesses have the ability to implement multi-modal mobile solutions, improving workflow, adding efficiencies and saving millions of dollars with the emerging flexible platforms (Caines, 2008). Though not universally implemented, 4G services offer greater speed and connectivity among other solid advancements that enhance mobility and reachability. Thus, this paper briefly reviews the history of devices used for SCM and ERP applications through movement to mobile devices, as well as suggesting considerations for fit and viability and other factors that influence the utilization of newer technologies.

\section{Early System Interfaces}

An Interface is either a tool or concept that refers to a point of interaction between components and is applicable at the level of both hardware and software; thus allowing a component, whether it be a piece of hardware such as a mobile phone or a piece of software such as an Internet browser, to function independently while using interfaces to communicate with other components via an input/output system and an associated protocols (Wikipedia).
Interfaces always act to communicate between components or devices but increasing the line between software and hardware is blurring. The history of devices used for SCM and ERP type systems, including the variations of Manufacturing Resource Planning (MRP) and MRP II, follows a pattern of either adopting existing, modification of or creation of newer technologies. Further, the mobility and reachability of technologies needed to service users is limited by the location and space availability of these same users using in the context of these ERP and SCM systems.

For instance, the introduction of keyboarding was faced with the dilemma of the most efficient means for typing in information as keyboards were the only way for users to input information. Review of those early text entry systems suggests two significant tradeoffs: potential efficiency and training time and device size and character-set size. (Ward, 2000) However, in the early days of any data entry, the focus of any keyboard was mainly to allow enough characters for all information to be displayed on the screen and then sent through a cable to the mainframe with no thought of wireless connectivity.

Though not used exclusively for SCM related and ERP systems, Telnet terminals were the traditional method for data entry into these systems up until the late 1970 's. As terminal interfaces were designed, consideration was placed on the screen layout design based on text location, readability, the ability to show $\mathrm{X} / \mathrm{Y}$ axes and cursor movement throughout the display. The Network Virtual Data Entry Terminal (NVDET) consisted of a keyboard and a rectangular display capable of generating all ASCII set characters with a number of function keys associated with "FN" subcommands. The interface provided a mechanism for defining screen fields with special attributes, including fields with brighter intensity, reverse video or blinking and protected mode. This provided a means for displaying "forms" to be filled in by users in the applications (Day, 1977). 
For those applications that required mainframe computing power, the interface for systems were the International Business Machine (IBM) 3270 and Digital Equipment Corporation (DEC) VT series of computer "dumb" terminals. Dumb terminals mean no processing power was in the terminal itself. These often had proprietary coding and controls which tied customers into use of the companies' hardware. In the case of the DEC VT, terminals were controlled by the manufacturer's own proprietary control codes or escape sequences, which were used to control things like cursor movement and deletion. By the late 1970's, American Nation Standards Institutes (ANSI) defined the standard for escape sequences (X3.64 standard). DEC complied and made its systems backward compatible to move from the legacy to industry standard. Subsequently, DEC also expanded its systems popularity by offering systems not only on its own platforms but in UNIX based applications which were becoming more popular for business applications. (DEC VT100, 2010). UNIX being a multi-tasking and multi-user operating system originally developed in 1969 by a group of American Telephone and Telegraph (AT\&T) employees working at Bell labs (Wikipedia).

For those companies who could not afford the expense of the IBM main-frame installations, IBM had introduced its System/3 computers in 1969. The IBM 5250 popular family of terminals was originally produced by IBM as a component of their System/3 system. The midrange System/3 offered a cheaper alternative to the larger, more expensive systems. The 5250 terminal was similar to its cousin the 3270 Information Display terminal. All of the system/3 systems, including those transitioned to IBM system 38, were all displaced by 1985 , with implementation of AS400 and other systems (IBM System/3). Up to this point in the history of SCM, MRP and ERP systems, there was not any concerted effort to be thinking mobile. Systems were large and required large staffs to maintain. Terminals and devices were directly connected to the systems to input data, extract data, process transactions and output standardized reports.

Companies soon learned that information needed to be timely, accurate, relevant, sufficient and cost effective to be useful in decision making. Overtime, firms began implementing a wide range of automated processes and employee procedures to ensure accurate information being entered into their databases. Bar-coding became the most wide-spread tool for automated data capture.

Bar coding is now a common means of managing inventory including the tracking of sub-assemblies used for manufacturing, tracking components utilized from the point of manufacture to actual use on the shop floor and tracking distributed products to the end consumer. Bar-coding captures the origin of the product, associates components and sub-assemblies to orders and acts as the mechanism for capturing additional data related to manufacturing processes llabor capture, functionality, reliability, maintainability, etc.). The bar-coding process can then be integrated with other systems, such as time and attendance, human resources and other systems not already integrated into the ERP system. Originally, bar-coding procedures required "manual" intervention by users to scan or place codes through readers. Today, this data is often "wired" directly through an interface to the system.

As PCs became more widely introduced after 1985 , more robust methods to access system solutions were implemented eliminating the need for dumb terminals on which legacy systems were based. Today we might call these thin clients. The PC solutions obviously gave end users the means to have a greater range of usability for in-house or packaged SCM, MRP and ERP solutions, as well as standard business applications, such as Microsoft products (eventually Office), Lotus and other burgeoning PC tools. Terminal emulators including Rhumba for Microsoft 
Windows based computers became available to allow companies to utilize one set of terminals (namely PCs) for their user interfaces. Subsequent versions of Windows and other operating systems soon began to make an emulator as a standard part of the product offerings. In the DEC realm of computer applications, terminal emulation software of VT100 and successors (particularly the VT220) became a staple of many products. Hyper Terminal was a default emulator in editions of Microsoft Windows up through and including Windows XP, the VT52, VT100 and various other emulations. Hyper terminal was capable of modem and telnet connection to hosts (DEC VT100, 2010).

It soon came to be that "hard-wired", dedicated terminal installations were not as cost-effective or efficient in terms of support, space and usability as the requirements of users wanted or needed for the future. So, the IT industry began thinking wireless, as did the SCM and ERP industries. Firms began looking at RF (radio frequency) data collection systems to transfer data from the shop floor directly into computer database systems without the limitations of wired equipment. Guided by the Institute of Electrical and Electronics Engineers (IEEE) standards, frequencies were made available through industry to standardize interfaces between manufactures and users. Typical installations included computers resembling laptop computers with embedded wireless controllers and adding protective coverings, such as those used on Panasonic Toughbook's for use in environmentally challenged locations (Mandal, 2002).

\section{Later Mobile Interface Considerations}

During the late 1990's, handheld devices came into use, initially as "stand alone" units as opposed to the integrated smartphone of the current day. Though adaptation of these devices continued worldwide, initially there was a requirement to attach modems and reading devices to capture and transfer data. There was not a single integrated device.
Devices from the mid-2000 era ranged from the PDA (Personal Digital Assistants), handheld PC, to other hybrid devices. At that time, Palm OS -based PDA's and eventually the Treo lines of phones were the predominant mobile devices in use. These handheld PC's utilized "smaller" versions of the more wide spread PC Windows's operating systems, such as Windows CE. Typical cell phones of the time had a screen resolution of about $150 \times 150$ pixels and between 4 to 12 lines of text, as well as limited keyboard functionality. More advanced smartphones provided larger screens with higher processor power, more memory, storage and the ability to utilize browsers. Of course, tremendous technological advancements in smartphone, tablets and similar devices, as well as the unprecedented impact of the iPad and related applications have expanded opportunities for business process improvement in the current era.

Since the introduction of the Apple iPad, even greater process improvements are possible using mobile computing. With its size, portability, memory storage, processing speed and flexible connectivity, this device brings new and greater opportunities for the future. The success of this device is its seamless interoperability with other devices and numerous applications that are accessed wirelessly almost anywhere.

In December 2010, UNIT4, a leader in ERP and financial management software, expanded its Agresso Business World's (ABW) dynamic reporting capabilities to both the Apple iPhone and iPad. This tool allowed for dynamic querying of database information, with the ability to create and store reports, using "drag and drop" functionality. According to Ton Dobbe, VP Product at UNIT4, "The Agresso VITA architecture is the foundation for our iPad/iPhone reporting capabilities, ensuring that any report or analysis is always in-sync with the organization's actual business model and structure - irrespective of where and how the data is presented. This provides 
huge value for users as they can securely access critical, relevant and accurate business data from their ERP system at any time, and respond to queries and external pressures quickly and effectively" (HT Syndication, 2010).

In July 2011, CA Technologies introduced its Project and Portfolio Management (PPM) solution, which will work with users of "Chatter" a popular sales force communication tool and private enterprise social network to allow project teams to collaborate and easily follow requirements and status updates on mobile devices like the iPad, iPhone or BlackBerry. PPM solutions such as Compuware "Changepoint" (http://www.compuware.com) allow firms to create a cohesive and consolidated understanding of their total business environment, utilize resources to make informed spending and resource management decisions and communicate their business needs to stakeholders. Further, PPM solutions allow organizations to management projects end to end while creating control over individual applications, products, investments and projects.

There are a number of competing tablet devices available to the general users in addition to the iPad, such as RIM (Blackberry), SAMSUNG (Galaxy) and other various Android operating system devices from Motorola and HTC. The key to evaluating any of these devices will be when SCM and ERP system vendors and internal developers spend their time and company resources to develop suitable applications for these devices.

Of course, smartphones present a smaller alternative to the tablets. Users already are carrying these devices as a means of communication and social interaction for both business and personal reasons. Whether or not applications can be effectively scaled to the size and power of these devices is still to be researched and applied where possible.
This trend toward accessing firm information remotely is continuing. Plans call for greater integration with leading ERP systems to bring in critical resource information to facilitate greater portfolio planning and decision-making. For ERP standouts, such as SAP and Oracle, pre-built adapters are available from CA Technologies and Pervasive Software to enable organizations to maximize the business intelligence from data investments they have already made (HT Syndication, 2011).

\section{Considerations for Use}

\section{The Supply Chain}

Supply chain management (SCM) is the management of a network of interconnected businesses involved in the ultimate provision of product and service packages required by end customers (Harland, 1996).Within mobile Supply Chain Management (mSCM), integration of real time events utilizing wireless technology enables a constant flow of up-to-date information horizontally and vertically inside and outside the organization. Since Supply Chain Management is concerned with information exchange and market response, real time events enhance supply chain performance. Real time information is necessary to reduce response lag time, delays in transaction processing and customer service and missed market opportunities such as order-todemand. Since prices of products and services can be changed utilizing dynamic pricing, mSCM helps companies to cope with changes of uncontrollable external factors affecting demand (Eng, 2006).

The key areas of impact for mSCM are in the areas of e-procurement, materials handling, inventory management, warehousing and logistics, fulfillment of orders, sales force and field automation as well as dispatch management. When these processes are integrated with a company ERP system, even greater functionality and process execution can be achieved. 
Further improvements in data collection are achieved by integrating employee and customer information with Geographic Information Systems (GISs) to leverage location and other aspects of company information complete with data scenarios so that subsequent business process management improvements can be undertaken. This in turn can reduce the costs associated with travel; improve productivity, process efficiency, data accuracy, service quality and employee mor ale (Doolin \& Al Haj Ali, 2008).

Regardless of the specific implementation of Supply Chain, ERP and MRP solutions, several key success factors must be kept in mind when implementing m-commerce solutions. Alexander Renz, as one example, lead RFID (radio frequency identification) program manager with Microsoft in Vedbaek, Denmark suggests that Business Integration, Business Optimization, Collaboration and Real world awareness are key success factors that must be addressed prior to beginning any $\mathrm{m}$-commerce project.

\section{Business Integration}

Successful business integration requires an adaptive foundation to be established in such a way that accurate, timely, relevant, sufficient and cost effective data be visible across end-to-end processes, which themselves are consistent and repeatable. Integration of internal processes are key in the context of implementing ERP systems, or other adaptations of MRP or MRO systems, to include basic supply chain management functionality that link "front- and back-office" functions. It is expected that Web portals be created utilized for information sharing.

\section{Business 0ptimization}

Successful optimization requires better decision making tools that allow for the greatest efficiencies in managing Business Process Management (BPM) applications. This can include Business Intelligence (BI) tools such as Microsoft BI Tools, SAP
Business Intelligence, ORACLE Enterprise BI Server, SAS Enterprise BI Server as well as other BI Analytics tools provided by a significant number of manufacturers.

\section{Collaboration}

Collaboration extends an organizations value chain across vertical processes to supply chain partners using networking and collaboration technologies such as Web portals and shared workspaces (like Microsoft SharePoint or Microsoft Groove). Such tools allow for the real-time, accurate exchange of information about demand and supply from suppliers and customers with the ability to quickly feed electronic orders into back-office and operational systems to shorten cycle times.

\section{Real-World Awareness}

It is essential that m-commerce application be rooted in a real world awareness of strategic implications and necessary process changes needed to operate in a dynamic global environment. Mobile applications, handheld devices, bar coding, global positioning systems (GPS) and RFID can all help obtain precise information about the movement of goods and services through the supply chain which helps cut time from order capture to fulfillment by providing real-time updates of data (including pricing and quantities) to leverage business opportunities (Haapaniemi, 2006). Yet, business processes and supporting information technology and systems must be adaptive to changing customer needs, governmental actions and technological trends.

Managing the supply chain with wireless technology is also a challenging conventional wisdom of how SCM can be implemented. Wireless access offers a geographical reach to support and enhance the association with logistics and delivery systems. Services can be requested at any location without the demand being initiated through a static or fixed portal. This challenges organizations to 
be ready to do business anytime and anywhere. It also creates a need to manage dispersed resources and generates the need to review organizational designs and hierarchies. Obviously, this necessitates successful change management processes throughout the organization (Eng, 2006).

In mSCM, wireless technology coupled with software applications, such as CRM systems can be used to deliver personalized services to users in the supply chain. The main purpose is to develop long lasting and profitable customer relationships. This personalization includes effective use of resources, such as filtering information to relevant users, personalizing services to customers, automating the sales force and creating opportunities to cross-sell related services based on customer purchase history. This approach to personalization not only offers instant services and user interfaces but also enabling execution of multiple tasks at remote locations (Ryals, 2001).

\section{ERP}

The considerations for ERP and MRP systems in the use of mobile devices and the associated benefits of wireless technology are not inherently different than those described for SCM. The main differences between ERP and SCM are the broader scope of the data being collected and analyzed along with a broader range of processes associated with ERP systems. Further, depending on where "sensitive data" is housed within systems, such as human resources and financial data, there may need to place more controls on both the server and client sides of the mobile applications to support the security needs of the organization.

\section{Fit, Viability and Organizational Acceptance}

Several studies suggest necessary conditions for mobile devices to successfully interface with SCM, ERP, MRP and related systems. A. K. Tjan (2001) introduced the concepts of fit and viability. Fit measures are defined as the extent to which applications meet the core competence, structure, value and culture of the organization. The idea is that any application must fit in with the way employees interact between themselves and their customers. A complementary attribute is viability. Viability measures the valueadded potential of new applications, requirements of human resources and the capital needs based on these applications (Liang, Huang, Yeh, \& Lin, 2007). Viability requires a firm to ask if this new application is worth the trouble and expense of implementing.

Fit and viability directly relate to the economic value of $\mathrm{m}$-commerce and the delivery of information to ERP and SCM systems within the firm (Liang T. P., 2002). Liang (2002) suggested that "reachability" and "mobility" are drivers of m-commerce. Having decided an application is worth implementing, that application must meet some minimum level of functionality and capability to actually be of use. Liang (2002) suggests that Product and Service Localization, Product Personalization, Ubiquity Enhancement, Instant Connectivity and Convenience enhance the mobility and reachability of $\mathrm{m}$-commerce applications.

Product and service localization is the process of adapting a product or a service to a particular language or culture with the desired local look-and-feel to be successfully used by customers and organizational users. Slightly different is the concept of personalization which involves using technology to accommodate the differences between users. Ubiquity enhancement is the notion that the ability exists to use applications everywhere and that products and services can be purchased anywhere at any time. Further, the IT architecture must be in place so that connectivity is a constant state for any user. Just as important, applications and devices must be convenient to use and not require any special information or access not already available. 
Success in the marketplace requires that all five of these attributes be in place.

A key aspect of evaluating these attributes of $\mathrm{m}$-commerce comes from awareness that the decision to purchase and implement $\mathrm{m}$ commerce applications requires not only acceptance and assimilation by customers but within the organization as well. That is, fit, viability, mobility and reachability of applications are success factors within organization but these same factors must be applied to the end users as well. They may not match up. This is not necessarily a new concept, but the implementation of $\mathrm{m}$ commerce interfaces begins with an approach based around requirements and the factors which collectively explain the reason for the technology innovation decision. Ultimately, there must be a time and cost benefit to someone.

As mentioned before, Product and service localization, Product personalization, Ubiquity enhancement, Instant connectivity and Convenience must be fit and viable as well as having a successful m-commerce implementation. This is influenced by 1) the perceived attributes of the technology innovation, 2) the evaluation of organizational characteristics, and 3) any environmental considerations (Doolin \& Al Haj Ali, 2008). While most organizations grasp the need to evaluate technology innovations, they fail to understand that the characteristics of the organization or the end-user may preclude a successful implantation of m-commerce. Some organizations do have the culture, leadership or structure to have success implementing m-commerce; some need to think about a change to their culture to make m-commerce a possibility. Further, there may also be environmental issues outside of the organization such as IT infrastructure, legal impediments or other factors that limit or prevent a firm implementing any or all aspects of an m-commerce implementation.

After conducting an expansive study of three different organizations, Bill Doolin, of
Auckland University of Technology, New Zealand, and his associates concluded that the decisions made by organizations to utilize mobile technology for Supply Chain applications was heavily influenced by the proactive and innovative attitudes of internal users toward IT and the history of IT use at each company. Leadership from the top-level down was essential to the success of the implementation. Where the executives were not involved in every decision, the success of the implementation required complete and unqualified support of the organization. They also found that there was a distinct difference between organizational readiness and user readiness. Though the IT department was fully ready and positive about the use of the technology, the lack of readiness from some key internal users tended to slow adoption and increase the time needed for full scale technology implementation (Doolin \& Al Haj Ali, 2008). Interestingly, user reluctance can be mitigated by forced implementation, particularly at the hands of mighty companies where the livelihood of subordinates or suppliers is acceptance voiced in a do or die proposition.

Wal-Mart has been an ultimate example of "mandating acceptance" in its implementation of technologies, particularly in its SCM implementation. Suppliers must abide by its technology initiatives in order to do business with the retail giant. In 2002, Wal-Mart suppliers needed to be part of the Retail Link network in order to place orders with Wal-Mart; in 2005, suppliers needed to be compliant with RFID technology to allow Wal-Mart to track its purchased merchandise from birth to grave (Sezen, 2006).

\section{Mobile User Interface Implementation}

The variety of mobile devices and operating systems becoming available on the market today can be challenging for organizations seeking to develop ubiquitous interfaces for each type of device. It is desired, yet not always possible, for companies to standardize the devices and applications 
used for the business applications. However, where standardization of devices and applications is not possible, organizations must select hardware and software that is flexible to work with. Mobile network suppliers, hardware manufacturers and technology consortiums are constantly working on increased stability, range and speed in data transfer rates through their products and services. Organizations must select technologies that have the best fit and viability for their organization while allowing for the most reach and mobility for users.

\section{Adaptation}

Adapting traditional desktop applications to mobile devices can be broken down into client-side, server-side and proxy based approaches. The key issue is transitioning a traditional Internet browsing screen down to a smaller device that has a smaller screen that allows for less information to be visible at any one time. One approach is to deliver PC content directly to the mobile device. The formatting of content on the PC device may involve delivering cascading "style sheets" directly to the mobile device; or, on the server-side, preparing the content for device delivery; or a proxy-based approach that address an intermediate stage for facilitating the adaption process (Butler, 2002). An alternative approach is to deliver content in a format designed for mobile devices, hence the recent success of smartphones and tablets. Kurbel, Jankowska and Nowakowski (2006) suggest before deciding which implementation approach to take, organizations and designers look at the needed mobile content, style and layouts, structure of the information being presented, implementation issues, business process impact, system integration, connectivity and security concerns (Kurbel, Jankowska, \& Nowakowski, 2006). Based on these implementation concerns, solutions must fit and be viable to the organization as well as being mobile and reachable for the users.

\section{Content}

Mobile applications should consider or minimizing unnecessary elements such as banners and images. The key is to focus on what is essential information with some sense of localization and personalization. Often times, the original application will have many extraneous boxes, lines, tabs or other "bells and whistles" that though somewhat appropriate for larger displays, are unsuitable for most mobile devices. Of course, where possible, considerations with iPads and recent tablet devices may make this task less daunting and more feasible to keep certain features that may add to the usability of the application. Regardless of the device, replacing banners and images with short textual descriptions assist in reducing the amount of data needing to be transmitted. If the original application has extraneous information that is not required by the mobile user, these bits of data should be considered for removal. However, organizations and designers must also work with a vision of the future in considering potential data elements to mitigate the need of future rework.

\section{Style and Layout}

Some elements of color or font size may provide value to display on PCs and various "wired" terminals, but again may not work well on the mobile device. Users expect devices to reflect product localization and custom personalization. Unnecessary breaks and margins can be removed to fit the limitations of the smaller display and data transfer requirements.

\section{Structural}

The flow of information can be adapted by splitting information into smaller fragments and displayed in multiple pages. This at times may be a challenge when the original application already has "too many" pages. 
As discussed earlier, it is possible that some of the fields found in the original application can be removed to allow a better flow of information on the mobile device. Data may also be arranged to display more critical data first, or by some order by selected data criteria. Thus, at some point, it may become more efficient to redesign the interface specific to a type of mobile device rather than tailoring an existing set of applications.

Ultimately, the goal is for the user not to have to actually type in much data. So, predefined lists, drop-downs, radio buttons, check boxes and the like can be utilized. Menus must be intuitive and the amount of browsing time reduced. Arranging data in a tree structure also facilitates improved navigation for the user interfaces, and the amount of text actually presented on the screen should be minimal to avoid much vertical and horizontal scrolling (Kurbel, Jankowska, \& Nowakowski, 2006). Additionally, voice activated commands and touch screens, where possible, allow users to keep their hands off keyboards and focus on the work to be done.

\section{Implementation}

Early in the design process, consideration should be given to how the mobile devices and applications are going to be introduced to customers and internal users. At some level, you would like the information being relayed from a mobile device to be no different from any other device. Information received would be treated no different than any other source, yet there still need to be internal users familiar with the strengths and weaknesses of mobile devices. Automating data entry for ERP systems can produce major returns for those companies spending millions of dollars on systems, which are only as good as that data that feeds them. Within the supply chain, technology advances allow for such things as using mobile devices to deliver a variety of applications, from scanning documents during receiving, to allow picking by voice command, to utilization of RFID at receiving docks, the ability to get the right person to the right job at the right time with right information is key and ultimately results in reduced labor costs (Shacklett, 2010).

\section{Business Process Impact}

Obviously, the implementation of mobile devices will impact business processes, not only from the location of where processes are being performed but in the timing and flow of processes currently in place to what processes will be in place in the future. It may not be necessary to redesign processes simply because a mobile capability exists; embedding mobile processes with existing processes can be a solution. Decisions based on process change must consider where the most benefits can be obtained from mobile technology deployment (Shacklett, 2010).

\section{System Integration}

Customers and users are expecting access everywhere, every time. They are expecting instant connectivity and a ubiquitous experience. Since most legacy and back-end systems are at various stages of maturity and have various database and platform dependencies, there may be a significant amount of time and expense to get the applications ready for mobile technology deployment. There may be additional technology initiatives along with needed cultural changes within the company that must be coordinated with any implementation to assure plans move forward in concert with corporate strategy.

\section{Security}

Lack of security is a possible threat to company internal systems, data and resources. The standard use of passwords and inclusion of biometrics, or GPS tracking can provide a number of benefits to an organization. Since people tend to value their mobile devices, there is a likelihood they would not share the device, and more likely to report them as missing. Protecting personal information by denying access to 
unauthorized users and recovering the device would be important.

Obviously, since the mobile devices can be lost, the company must have the ability to remotely monitor the device and shut it down when necessary (Shacklett, 2010). In a method similar to how banks can tell when someone is using a credit card in unusual locations and can take steps immediately, the same can be applied to mobile device tracking. In a recent study performed by McAfee and Carnegie Mellon University, it was surprising that companies were not often using" location-aware" products to protect their data. Although behavior monitoring combined with locationawareness can significantly strengthen mobile security, it was found only 22 percent of users are using location-awareness and that 30 percent are not considering using it (Rashid, 2011).

A key to addressing concerns over security is to have a clear company security policy which is followed by employees. Education and training for users and customers enhance the success of these security policies. In a McAfee and Carnegie Mellon University study, it was found that one of three employees and two-thirds of the employees were not aware of their organization security policies, even though 95 percent of companies have mobilesecurity policies in place to protect their enterprise data. Most of the companies reported their employees do not understand how permissions and other access settings on their mobile devices work. Many employees did not back up data regularly, regularly change passwords and pins and inappropriately stored company-sensitive data on their mobile devic es (Rashid, 2011).

\section{Bandwidth and Connectivity}

Any effective system using mobile technology must be ubiquitous, convenient and connected to an infrastructure that allows applications to be personalized and localized. This arrangement results in greater confidence in the availability and reliability of data, so that users can make correct and timely decisions.

In the realm of Supply Chain and ERP related system applications, customers and suppliers within a firm value chain are dependent on real-time information to support and react to information business relationships require. The concept of "anytime, anywhere and anyway" has been the prevalent theme of converging multi-service networks of diverse technologies in recent years. Known as "Always Best Connected" or ABC, the most recent unifying network solutions are the Fourth Generation (4G) communication networks (Antoniou, 2007).

Within the 4G landscape are competing technologies implemented by wireless providers who are constantly in competition to be the fastest and most reliable network. These new technologies offer higher throughputs, lower cost-per-bit of information and are more "friendly" to IP traffic. Long-Term Evolution (LTE) technology will have a projected 303.1 million, compared to 33.4 million for (Wireless interoperability for microwave access) WiMAX by 2014. WiMAX is currently the leader in $4 \mathrm{G}$ deployments but LTE is supported by most of the leading wireless operators worldwide. LTE offers peak download rates of $150 \mathrm{MBS}$ and 50 MBS peak upload rates, and provides substantially improved end-user throughputs, sector capacity and reduced user plane latency, bringing significantly improved user experience with full mobility and making possible for even more devices to utilize peak wireless performance. WIMAX is an IP based, wireless broadband access technology with performance similar to $802.11 / \mathrm{Wi}-\mathrm{Fi}$ networks, intended for wireless "metropolitan area networks". WiMAX can provide broadband wireless access (BWA) up to 30 miles for fixed stations, and 3 - 10 miles for mobile stations, in contrast to WiFi/802.11 wireless local area network limited in most cases to only 100 - 300 feet. (WIMAX.com Broadband Solutions, 2011). 


\section{Cloud Computing}

Cloud computing is a marketing term used by third party technological providers to describe a utility package that delivers computing as a service rather than as a product. Shared resources, software applications, data access/retrieval and information storage, etc., are provided to networked computers without the user knowing the location or architecture of the computing infrastructure. Users may pay for a standardized set of technologies common to all business users or pay for more capabilities. In this contest, they do not need to worry where their information comes from, how it is generated or how it is transported. They simply pay a bill for the services they use.

Recent problems within the global economy have further encouraged companies to look for more efficient ways to manage their supply chain. Many manufacturing companies have signed up for pay-as-you-go SRM models to access logistic services via the Cloud. Such services allow these companies to find easier and more efficient business solutions to effectively communicate with their suppliers, share information with transportation providers and end users (Hickey, A.R., 2010). Services provided through the cloud not only help companies to have access to technologies that they are unable to maintain by themselves, but also they may have a strategic advantage by under-cutting sunk costs of companies tied to existing architectures and older technologies. By using cloud computing, companies share costs with other participants in the network. Cloud computing allows companies to find virtual low cost suppliers and outsource the supply chain activities to them. Outsourcing of such activities will allow companies to focus more on their primary activity. While supply chain technologies are expensive to build and maintain and are a competitive advantage for those who have them, cloud computing offers an affordable solution (Hickey, A.R., 2010).
The cloud technology blurs company boundaries, since companies are not competing individually against other companies, but rather supply chains are competing against each other. Cloud computing has changed the relationship between companies which participate in the same supply chain. They are sharing much more information within the chain related to their sales, and they have integrated their systems within each other whereby they have increased the transparency of their transactions. The importance of cloud computing and the management of supplychain relationship is reflected in the boardroom by the number of firms adding senior supply-chain positions on par with traditional COO, CFO, etc management positions (Hickey, A.R., 2010).

Companies need to consider the challenges that cloud computing may bring to their supply chain managers (Schramm et al., 2011). Cloud computing may increase the pressure on the supply chain by increasing the speed of delivery to market for new products and services; forcing companies to re-invent the supply chain to be more dynamic, scalable and competitive; and requiring ongoing complex discussions and decisions impacting all supply chain partners to adopt, change or not accept cloud computing alternatives. At stake for some firms is control over information. Firms risk loss of sensitive information and intellectual property, etc. based on a false sense of trust with firms participating in their value chain. These decisions are not made lightly.

The future of cloud computing grows while the prices of iPhones and mobile devices continue to drop. Cloud computing allows more suppliers to participate within a value chain regionally, nationally and globally, possibly providing more competition. By using mobile devices and applications, any firm can track products, optimize delivery routes, make decisions while using real-time data, etc. 
And at the same time, mobile devices have become more powerful, have more storage and more graphics possibilities. In a recent study published by ABI Research, cloud services will support more supply chain activities and enterprise data. This study found that more than 240 million businesses will use cloud services via mobile devices and they will expand the enterprise applications used in such devices by 2015 (Koblentz, 2010).

\section{Cost Considerations}

Implementation of the newer ERP, SCM and Cloud computing technologies varies depending on the number of devices, the expected bandwidth, current company infrastructure, IT technology strategies, vendor relationships and the like. Costs not only include hardware but the expense of programmers, integration costs and developing a support network for the implementation.

Firms must consider the costs of modification to existing applications and new applications. These costs include internal and external programming resources needed to scope, design, test and implement applications into production ready environments. Business and end-user time must be considered and planned for as well as that of the technology department to plan and coordinate the implementation and support of the end solution.

There are the costs of on-going support including specific support requirements for individual applications, internal resource support or the software support for vendors including ongoing training. This may even involve third-party resources specifically responsible for support of the particular version of the software, and coordinating any support requirements of internal IT or customer relationship management. Organizations may need to consider adding or expanding help centers. Specific servers may be required to support the dedicated needs of the wireless implementation as well as those to support the applications alone.

There are costs to maintain on-going partner relationships depending on specific implementations of the mobile technology. There may be effects on how partners utilize the technology and applications, particularly for those involved in the supply chain, where customers and vendors are often tied in to specific applications for monitoring company inventory, pricing, buying consortiums and the like. Costs may be absorbed differently by different partners.

Costs of handheld devices will need to be monitored as devices and capabilities become more ubiquitous. There may be a time when specified devices can be replaced with off-the-shelf hardware. Because of the increasing number of devices and features of devices as well as different operating systems, users will see an endless number of combinations of devices making standardization of devices difficult. Users should demand common interfaces and architectures, allowing users the choice of any hardware/software combination and the ability to select any application. There are hundreds of smart phones and tablet offerings to utilize the growing number of wireless network capabilities. However, the discussion of the devices obviously can be expanded to netbooks and other more compact versions of PCs depending on the users' physical environment. Considerations must also be given to the number of "back up" units to assure on-going, consistent availability of devices.

Successful wireless implementations require a technology infrastructure capable of supporting and organizations fit and vitality. A changing environment, vendor relationships, contracted services and many combinations of internal and external infrastructure possibilities make costing difficult. Control of cost dictates that firms plan for the applications and services they would like now and in the future and the build architecture to meet these needs. It is 
often problematic to build architecture and then wait for users to figure out how to use it.

Any organization considering implementation of hand-held or mobile devices will need to go through an entire process of evaluating costs against the benefit of the implementation. Of course the very nature of Supply Chain Management necessitates the need for timely, accessible information, often at customer and off-site locations. Requirements gathering, involving required and "like to have" considerations will need to occur, followed by analysis and design and all the normal components of a Systems Design Life Cycle (SDLC) analysis, even though the same exercises may have occurred for the original business applications themselves.

\section{Conclusion}

Implementing mobile devices particularly in regard to SCM and ERP related systems requires thoughtful introspection with regards to fit and viability of a specific firm. Further, the reach and mobility needed to fit specific business needs must be considered. Many different types of devices have been used over the last half century related to these systems. As the modern world becomes increasingly mobile, so have the needs of users competing in the global marketplace. Recent upheavals in the business cycle make acquiring resources to go mobile more difficult, but at the same time finding resources to go mobile may be essential to compete effectively as firms want to be in position to react to positive business climates. Arguing for funds to support mobile wireless technologies may be viewed as risky in a down business cycle but historical trends argue in favor of mobile technologies. In this context, understanding what is required to implement $\mathrm{m}$-commerce effectively will save resources and strengthen arguments to acquire the necessary resources to move forward.

It is not readily apparent that there have been full m-commerce implementations of
SCM, MRP, MRO, or ERP related systems on to mobile or hand-held devices, although there has been a great deal of thought into how this could occur. There have been limited mobile implementations of ERP solutions, mainly in reporting tools. There are also some industry specific examples such as cockpit data capture of data feeds within airline systems, on "truck" delivery information in the trucking industry as well as a "good deal" information for sales force related implementations. Vendors offer the "promise" of more full-fledged opportunities but often look for "partners" in the development efforts to support the cost and specific pursuits of the desired end results. Some caution is warranted. Therefore, the conclusion of this paper points to a multifaceted approach in examining any solution when transitioning to mobile devices, realizing the hardware or devices themselves are not the specific end result, but rather the entire effort of all things needed to support the delivery of applications, data and timeliness delivery to these devices.

\section{References}

Antoniou, J. \& Pitsillides, A. (2007). "4G Converged Environment: Modeling Network Selection as a Game," Mobile and Wireless Communications Summit 2007, 16th IST (15). Budapest: IEEE.

Butler, M., Giannetti, F., Gimson, R. \& Wiley, T. (2002). "Device independence and the Web," IEEE Internet Computing, 6(5), 81-86.

Caines, R. (2008). 'Next Major Mobile Wiereless Market: Industrial,' Material Handling Management, 35.

Day, J. D. (1977). Telnet Data Entry Terminal Option.

DEC VT100. (2010). Retrieved August 2, 2011, from GreenScreen: http://terminalemulators.net/2010/07/decvt100/

Doolin, B. \& Al Haj Ali, E. (2008). "Adoption of mobile technology in the supply chain: an 
exploratory cross-case analysis," (I. Lee, Ed.) International Journal of e-Business Research, 4(4), 1-15.

Eng, T.- Y. (2006). "Mobile supply chain management: Challenges for implementation," Technovation, 26(5-6), 682-686.

ERP History. (2007). Retrieved 2 2011, August, from ERP and More: http://www.erpandmore.com/erpreference/erp-history/

Gazis, V., Houssos, N., Alonistioti, N. \& Merakos, L. (2003). "On the Complexity of "Always Best Connected" in 4G mobile networks," in. 2003 IEEE 58th Vehicular Technology Conference (2312 - 2316). Orlando: IEEE.

Haapaniemi, P. (2006). 'Supply chain makeover,' Retrieved June 16, 2011, from Microsoft: Midsize Business Center: http://www.microsoft.com/midsizebusiness /business-leaders/business-process/supplychain-management.mspx

Harland, C. M. (1996) 'Supply Chain Management, Purchasing and Supply Management, Logistics, Vertical Integration, Materials Management and Supply Chain Dynamics,' In: Slack, N (ed.) Blackwell Encyclopedic Dictionary of Operations Management. UK: Blackwell.

Hickey, A. R. (2010). Report: Mobile Cloud Computing A $\$ 5$ Billion Opportunity, accessed at: http://knowledge.wharton.upenn.edu/articl e.cfm?articleid=2669

HT Syndication. (2010). UNIT4 Launches Mobile App to Support Dynamic Reporting. The Pak Banker, Lahore, 1.

HT Syndication. (2011). CA Technologies PPM Solution Integrates Salesforce.com Chatter to Meet Business Demand for Collaboration. The PAK Banker, Lahore.
IBM System/3. (n.d.). Retrieved August 2, 2011, from ibm.com: http://www03.ibm.com/ibm/history/exhibits/rochester /rochester_4008.html

Koblentz, E. (2010) ABI Research: Mobile Cloud Computing Reaches \$5.2B Market by 2015. Accessed at http://mobileenterprise.edgl.com/topstories/ABI-Research--Mobile-CloudComputing-Reaches-\$5-2B-Market-By201560640

Kurbel, K., Jankowska, A. M. \& Nowakowski, K. (2006). "A mobile user interface for an ERP system," Issues in Information Systems, VII(2), 146-151.

Liang, T. P. (2002). 'Desicion Support Systems and Business Intelligence,' Tapei: Best Publishing Co.

Liang, T. P., Huang, C. W., Yeh, Y. H. \& Lin, B. (2007). "Adoption of Mobile Technology in Business: A Fit-Viability Model," Industrial Management \& Data Systems, 107(8), 11541169.

Mandal, P. \& Gunasekaran, A. (2002). "Application of SAP R/3 in on-line inventory control," International Journal of Production Economics, 47-55.

Rashid, F. Y. (2011). "Mobile Device Data Losses Pose Rising Security Risk: Survey," Retrieved August 3, 2011, from IT Security and Network Security News: http://www.eweek.com/c/a/Security/Mobil e-Device-Data-Losses-Pose-Rising-SecurityRisk-Survey-515021/

Ryals, L. \& Payne, A. (2001). "Customer Relationship Management in Financial Services: towards information-enabled relationship marketing," Journal of Strategic Marketing, 9(3), 3-11.

Schramm, T., Nogueira, S. \& Jones, D. (2011). "Cloud Computing and Supply Chain: A Natural Fit for the future," 
http://www.logisticsmgmt.com/article/clou d_computing_and_supply_chain_a_natural_fit_ for_the_future/

Sezen, B. (2006). 2-Wal-Mart Supply Chain. Retrieved April 16, 2011, from gyte.edu.tr: www.gyte.edu.tr/dersler/546/.../2-WalMart\%20Supply\%20Chain.ppt

Shacklett, M. (2010). "Mobile technology and the supply chain," World Trade 100, 22-26.

Shankar, V. O. \& Driscoll, T. (2002). "How Wireless Networks Are Reshaping the Supply chain," Supply Chain Management Review, 6(4), 44-51.

Walsh, K. \& Johnson, J. (2008). “Challenges Facing Front Ends for 3G and 4G Multimode Handsets," Microwave Journal, 28-31.

Ward, D. J., Blackwell, A. F. \& MacKay, D. J. C. (2000). "Dasher a Data Entry Interface Using Continuous Gestures and Language Models," UIST '00 Proceedings of the 13th annual ACM symposium on User interface software and technology (p. 1). New York: ACM.

WIMAX.com Broadband Solutions. (2011). "What is WIMAX?," Retrieved August 4, 2011, from WIMAX.com: http://www.wimax.com/general/what-iswimax

Zahariadis, T., Grüneberg, K. \& Celetto, L. (2010). "Seamless Content Delivery over Mobile 3+/4G Networks," Mobile Networks and Applications, 16(3), 351-360. 